\title{
Métodos para estimar a forragem consumível em pastagem de capim-elefante ${ }^{(1)}$
}

\author{
Antônio Carlos Cóser(2), Carlos Eugênio Martins ${ }^{(2)}$, Fermino Deresz(2), Ary Ferreira de Freitas(2), \\ Domingos Sávio Campos Paciullo ${ }^{(3)}$, José Augusto Salvati( ${ }^{(2)}$ e Lilian Tavares Schimidt(4)
}

\begin{abstract}
Resumo - O objetivo deste trabalho foi avaliar a eficiência e a confiabilidade de métodos para estimar a forragem consumível em pastagem de capim-elefante (Pennisetum purpureum Schumach.). Foram usados o método do rendimento visual comparativo (MRVC) e o método do pastejo simulado (PSIM). Foram realizadas amostragens em 20 de março e 5 de abril de 2001, com um e quatro observadores para PSIM e MRVC, respectivamente. As estimativas obtidas foram analisadas por meio de regressão linear da produção de matéria seca em razão dos padrões estimados e as médias comparadas pelo teste $\mathrm{F}$, a $1 \%$ de probabilidade. Não houve diferença entre os métodos. Tanto o MRVC como o PSIM podem ser recomendados para estimar a forragem potencialmente consumível em pastagem de capim-elefante. $\mathrm{O}$ treinamento dos avaliadores torna-se decisivo, especialmente quando o MRVC estiver sendo utiliza-
\end{abstract} do.

Termos para indexação: Pennisetum purpureum, alimentação animal, pastejo.

\section{Methods to estimate edible forage in an elephant grass pasture}

\begin{abstract}
The objective of this work was to evaluate the efficiency and reliability of methods to estimate edible forage dry matter on an elephant grass pasture (Pennisetum purpureum Schumach.). Visual comparative yield method (VCYM) and hand plucking (HP) were used. Two samplings, one in March 20, and another in April 5, 2001, with one and four observers for HP and VCYM, respectively, were done. Estimates of edible forage dry matter were analysed by linear regression and the average estimates were compared by using $\mathrm{F}$ test with a probability level of $1 \%$. There was no difference between the two sampling methods. Both, VCYM and HP were efficient to estimate potentially edible forage in the elephant grass pasture. The observers training is decisive, especially when VCYM is used.
\end{abstract}

Index terms: Pennisetum purpureum, animal feeding, grazing.

\section{Introdução}

O elevado potencial de produção do capim-elefante (Pennisetum purpureum Schumach.) evidencia a importância desta espécie para a produção animal (Alencar, 2002; Martins et al., 2002). No entanto, sua alta produtividade na estação chuvosa e a redução do crescimento na época seca resultam em vari-

\footnotetext{
(1) Aceito para publicação em 9 de abril de 2003.

(2) Embrapa-Centro Nacional de Pesquisa de Gado de Leite (CNPGL), Rua Eugênio do Nascimento, 610, CEP 36038-330 Juiz de Fora, MG. E-mail: acoser@cnpgl.embrapa.br, caeuma@cnpgl.embrapa.br, deresz@cnpgl.embrapa.br, ary@cnpgl.embrapa.br

(3) Embrapa-CNPGL. Bolsista do CNPq. E-mail: dscp@terra.com.br

(4) Rua Sagrada Família, 495, Bela Vista, CEP 45995-000 Teixeira de Freitas, BA. E-mail: liorural@bol.com.br
}

ações nas características morfológicas e nos teores de matéria seca, dificultando a utilização de métodos de amostragem, que devem ser sensíveis nessas duas condições (O'Rourke et al., 1984).

$\mathrm{O}$ peso da forragem torna-se importante medida de crescimento, pois permite calcular a taxa de lotação e interpretar o rendimento animal (Estrada et al., 1991). Contudo, a estimativa da forragem disponível é freqüentemente associada a alto erro experimental, podendo variar bastante entre os métodos e os observadores (Aiken \& Bransby, 1992).

O método direto do corte da forragem geralmente proporciona maior precisão que outros métodos de avaliação (Haydock \& Shaw, 1975). No entanto, para áreas extensas de pastagens, fornece apenas uma estimativa pobre de seu rendimento, principalmente quando a variabilidade de produção dentro da pastagem é grande e o aumento no número de amostras é inviável. 
Uma vantagem dos métodos indiretos é que a alta variabilidade encontrada nas amostras pode ser compensada com um maior número de amostras, aumentando a precisão do trabalho, em razão da facilidade e rapidez que esses métodos apresentam (Haydock \& Shaw, 1975).

O treinamento de avaliadores é de suma importância na recomendação e validação de um método de amostragem, sobretudo em pastagem com forrageira de crescimento cespitoso. Coelho (1984) relata existir tendência de superestimar estandes que possuam altura mais elevada e baixa densidade e, ao contrário, subestimar os que possuem menor altura e maior densidade.

Medidas como a altura da planta e a cobertura do solo, bem como as estimativas visuais, podem permitir melhor avaliação da produção de forragem em áreas sob pastejo, reduzindo custo, tempo e mão-deobra nas avaliações. Nesse sentido, Abramides et al. (1982) verificaram que a altura da vegetação é uma técnica viável para estimar a quantidade de forragem, em gramíneas que exibem hábito de crescimento prostrado. Por sua vez, Abramides \& Franzini (1981) observaram que, além da altura, a cobertura do solo é importante por possibilitar maior confiabilidade na estimativa da forragem disponível em gramíneas de crescimento cespitoso.

Baseados na técnica de dupla amostragem, Haydock \& Shaw (1975) desenvolveram o método do rendimento visual comparativo para estimar a produção de matéria seca de pastagens exclusivas de gramíneas. Neste caso, a avaliação da produção é feita a partir da alocação de quadrados de referência pré-selecionados, que constituem uma escala de rendimentos; é um método bastante preciso, usa técnicas não-destrutivas e possibilita maior número de estimativas de rendimento; sua precisão é função da calibração dos quadrados de referência. A diferença entre o método de Morley et al. (1964) e o de Haydock $\&$ Shaw (1975) é que o primeiro estima a produção diretamente no quadrado e o segundo relaciona a produção de um quadrado com a calibração dos quadrados de referência. Para Lopes et al. (2000), este é o método mais apropriado para avaliar pastagem de capim-elefante, mesmo superestimando a produção de forragem. Por sua vez, a técnica do pastejo simulado para estimativa da forragem potencialmente consumível em pastagens de capim-elefante, preconizada por Aroeira et al. (1999), é recente e tem sido usada nos experimentos de pastejo envolvendo essa forrageira.

O objetivo deste trabalho foi avaliar a eficiência e a confiabilidade de métodos na estimativa da forragem potencialmente consumível em pastagem de capim-elefante.

\section{Material e Métodos}

No campo experimental da Embrapa-Centro Nacional de Pesquisa de Gado de Leite, Coronel Pacheco, MG, foram realizadas duas amostragens ( 20 de março e 5 de abril de 2001) para estimativas da forragem potencialmente consumível pelos animais em pastagem de capim-elefante manejadas em sistema rotativo com três dias de pastejo e 30 dias de descanso, usando-se dois métodos de avaliação.

No método do rendimento visual comparativo (MRVC) foram determinados os padrões 1 e 3 , com a menor e a maior quantidade de forragem disponível dentro da pastagem, respectivamente, por meio de avaliações visuais de altura e densidade. Na sequiência, foi definido o padrão 2 (intermediário), e o padrão 0, em área com ausência total de forragem. Após a definição dos padrões e o treinamento dos quatro avaliadores, realizaram-se as avaliações visuais, individualmente, em cada uma das 25 amostras que foram coletadas manualmente e pesadas, usando-se amostragem do tipo sistemática, a cada três metros, com quadrados de um metro de lado, com um dos lados aberto para facilitar a amostragem. As amostras eram constituídas do componente folhas verdes provenientes de perfilhos aéreos e basais e as folhas senescentes e mortas eram descartadas. De cada amostra foi retirada uma subamostra, encaminhada ao laboratório para determinação de matéria seca. Depois desta etapa, cada observador realizava 30 novas avaliações visuais dentro da área experimental, em diferentes pontos, as quais foram convertidas em estimativas de matéria seca, a partir das equações de regressão da produção de matéria seca em função dos 25 escores de rendimento registrados por observador. 
O método do pastejo simulado (PSIM) preconizado por Aroeira et al. (1999) foi usado para a seleção de três touceiras de capim-elefante, representando as produções de forragem alta (3), média (2) e baixa (1) existentes na pastagem um dia antes da entrada dos animais em cada um dos seis piquetes avaliados. De cada uma dessas touceiras (amostras) foram removidas manualmente as folhas e colmos macios, simulando o pastejo. As amostras foram secadas em estufa, para determinação da matéria seca. Após a saída dos animais dos piquetes, foi contado o número de touceiras existentes numa área de $49 \mathrm{~m}^{2}(7 \times 7 \mathrm{~m})$ de cada piquete. A produção de matéria seca de cada amostra, em cada piquete, em cada amostragem, multiplicada pelo número de touceiras por piquete, resultou na estimativa da produção de matéria seca (kg/ha), originando 18 estimativas, em cada amostragem. Essas estimativas foram analisadas por meio de regressão linear da produção de matéria seca em função dos respectivos padrões estimados, usando-se o pacote estatístico PROC REG versão 8.2 para Windows (SAS Institute, 2001). As estimativas da forragem de capim-elefante consumível obtidas pelos dois métodos foram comparadas pelo teste $\mathrm{F}$ a $1 \%$ de probabilidade. Da mesma forma, os valores de intercepto $(\alpha)$ e do coeficiente angular $(\beta)$ das equações de calibração dos dois métodos foram comparados por meio do LSMEANS versão 8.2 para Windows (SAS Institute, 2001), a 1\% de probabilidade.

\section{Resultados e Discussão}

Os métodos de estimativa da forragem potencialmente consumível em capim-elefante sob pastejo apresentaram valores de coeficiente de determinação $\left(\mathrm{R}^{2}\right)$ iguais ou superiores a 0,92 no MRVC e de 0,83 no PSIM, na primeira amostragem, e valores de $\mathrm{R}^{2}$ mais baixos na segunda amostragem no MRVC (Tabela 1). Contudo, esses valores foram superiores ao mínimo preconizado de 0,80 de $\mathrm{R}^{2}$ para sua aceitação como método da avaliação de pastagem de capim-elefante, atendendo à premissa básica estabelecida. Portanto, ambos os métodos podem ser utilizados na avaliação da forragem disponível, embora o MRVC tenha permitido valores de $\mathrm{R}^{2}$ mais elevados quando comparado com o PSIM, nas duas avaliações. Os altos valores de $\mathrm{R}^{2}$ significam que uma proporção significativa da variação em produção de forragem potencialmente consumível pôde ser explicada pelos escores e padrões determinados pelos dois métodos de avaliação.

Valores de $\mathrm{R}^{2}$ entre 0,80 e 0,85 foram obtidos por Cóser et al. (2000) ao avaliarem a disponibilidade de forragem de capim-elefante manejado em pastejo rotativo com três dias de pastejo e 30 dias de descanso, utilizando índice altura da planta x cobertura do solo. Segundo Thomson (1986), valores de $\mathrm{R}^{2}$ inferiores a 0,75 são considerados insatisfatórios para predição da forragem disponível em pastagens; Cóser et al. (2002) preconizam valores de $\mathrm{R}^{2}$ iguais ou superiores a 0,80 para que o método seja recomendado. Tais avaliações eram realizadas anteriormente pelo método direto (corte) que, além de trabalhoso e destrutivo, era mais demorado e apresentava, em geral, maior variação, chegando a atingir valores de coeficiente de variação duas vezes superior aos obtidos com os métodos acima referidos (Cóser et al., 2000). Cóser et al.(1989) observaram altos coeficientes de variação na avaliação, sob cortes, de pasta-

Tabela 1. Equações de regressão, coeficientes de determinação $\left(\mathrm{R}^{2}\right)$ e coeficiente de variação $(\mathrm{CV})$ da produção de matéria seca em função dos escores estimados, em duas amostragens (20 de março e 5 de abril de 2001), usando-se o método do rendimento visual comparativo (MRVC) e o método do pastejo simulado (PSIM), por quatro e um observadores, respectivamente, em pastagem de capim-elefante.

\begin{tabular}{|c|c|c|c|}
\hline Observador & Equação de regressão & $\mathrm{R}^{2}$ & $\mathrm{CV}(\%)$ \\
\hline & \multicolumn{3}{|c|}{ MRVC - 20/3/2001 } \\
\hline 1 & $Y=256,85+763,50 X$ & 0,96 & 6,0 \\
\hline 2 & $Y=109,94+820,69 X$ & 0,95 & 7,4 \\
\hline 3 & $\mathrm{Y}=92,97+819,17 \mathrm{X}$ & 0,92 & 9,2 \\
\hline \multirow[t]{2}{*}{4} & $Y=138,16+815,81 X$ & 0,94 & 8,0 \\
\hline & \multicolumn{3}{|c|}{ PSIM - 20/3/2001 } \\
\hline 1 & $Y=-238,83+1046,38 X$ & 0,83 & 21,8 \\
\hline & \multicolumn{3}{|c|}{ MRVC - 5/4/2001 } \\
\hline 1 & $Y=-32,24+1.281,76 X$ & 0,89 & 11,3 \\
\hline 2 & $\mathrm{Y}=270,19+1 \cdot 140,14 \mathrm{X}$ & 0,89 & 12,0 \\
\hline 3 & $\mathrm{Y}=159,43+1.229,18 \mathrm{X}$ & 0,87 & 15,8 \\
\hline \multirow[t]{2}{*}{4} & $\mathrm{Y}=268,79+1.138,06 \mathrm{X}$ & 0,89 & 12,8 \\
\hline & \multicolumn{3}{|c|}{ PSIM - 5/4/2001 } \\
\hline 1 & $Y=-172,67+1.049,94 X$ & 0,83 & 21,1 \\
\hline
\end{tabular}

Pesq. agropec. bras., Brasília, v. 38, n. 7, p. 875-879, jul. 2003 
gens naturais em que a diversidade da vegetação era muito grande. Verificou-se, ainda, que no presente trabalho o MRVC apresentou menores valores de coeficiente de variação, de $6,0 \%$ a $9,2 \%$ e de $11,3 \%$ a $15,8 \%$, do que o PSIM, de $21,8 \%$ e $21,1 \%$, na primeira e na segunda amostragens, respectivamente.

O modelo de regressão linear foi o que melhor se ajustou aos dados, embora fossem testados outros modelos, como o quadrático, o cúbico e o exponencial.

Não houve diferença $(\mathrm{P}>0,05)$ entre os observadores no método MRVC nem entre os métodos de estimativa estudados, demonstrando que estes permitem estimar a forragem disponível de maneira semelhante (Tabela 2). Na segunda amostragem, realizada em abril, houve aumento significativo na forragem potencialmente consumível, fato este provavelmente relacionado às condições climáticas favoráveis ao crescimento do capim-elefante nessa época. A grande vantagem do método PSIM, do ponto de vista prático, é a necessidade de menor tempo de treinamento para sua execução. Com base nos resultados do presente trabalho, tanto o MRVC como o PSIM podem ser recomendados para avaliações da forragem potencialmente consumível pelos animais em pastagem de capim-elefante.

Em geral, tanto os valores de intercepto $(\alpha)$ quanto os de coeficiente angular $(\beta)$ foram influenciados pelas duas amostragens, sendo mais elevados na segunda (Tabela 3). Essas variações podem estar relacionadas com a estrutura do dossel vegetativo, principalmente por causa das modificações causadas pelas condições climáticas em cada amostragem, bem

Tabela 2. Médias estimadas da produção de forragem potencialmente consumível (base de matéria seca - kg/ha) e respectivos desvios-padrão, em duas amostragens (20 de março e 5 de abril de 2001), usando-se o método do rendimento visual comparativo (MRVC) e o método do pastejo simulado (PSIM), por quatro e um observadores, respectivamente, em pastagem de capim-elefante.

\begin{tabular}{cccc}
\hline Observador & $\begin{array}{c}\text { 20/3/2001 } \\
\text { (média } \pm \text { desvio-padrão) }\end{array}$ & $\begin{array}{c}5 / 4 / 2001 \\
\text { (média } \pm \text { desvio-padrão) }\end{array}$ \\
\hline \multicolumn{3}{c}{ MRVC } \\
1 & $1.663 \pm 93,8$ & $2.146 \pm 286,3$ \\
2 & $1.551 \pm 115,4$ & $2.138 \pm 320,5$ \\
3 & $1.535 \pm 143,3$ & $2.192 \pm 356,7$ \\
4 & $1.592 \pm 124,1$ & $2.230 \pm 316,2$ \\
\hline & & PSIM \\
1 & $1.754 \pm 404,2$ & & $2.027 \pm 407,2$ \\
\hline
\end{tabular}

Tabela 3. Valores de intercepto $(\alpha)$ e do coeficiente angu$\operatorname{lar}(\beta)$ por observador, das equações de calibração do método do rendimento visual comparativo (MRVC) e do método do pastejo simulado (PSIM), em pastagem de capim-elefante, em duas amostragens (20 de março e 5 de abril 2001) $)^{(1)}$.

\begin{tabular}{crrrrr}
\hline Observador & \multicolumn{2}{c}{ Intercepto $(\alpha)$} & & \multicolumn{2}{c}{ Coeficiente angular $(\beta)$} \\
\cline { 2 - 3 } \cline { 3 - 5 } & $20 / 3 / 2001$ & $5 / 4 / 2001$ & $20 / 3 / 2001$ & $5 / 4 / 2001$ \\
\hline \multicolumn{5}{c}{ MRVC } \\
1 & $256,85 \mathrm{~b}$ & $-32,34 \mathrm{a}$ & $763,50 \mathrm{a}$ & $1.281,76 \mathrm{~b}$ \\
2 & $109,94 \mathrm{a}$ & $270,19 \mathrm{~b}$ & & $820,69 \mathrm{a}$ & $1.140,14 \mathrm{~b}$ \\
3 & $92,97 \mathrm{a}$ & $159,43 \mathrm{~b}$ & $819,17 \mathrm{a}$ & $1.229,18 \mathrm{~b}$ \\
4 & $138,16 \mathrm{a}$ & $268,79 \mathrm{~b}$ & $815,81 \mathrm{a}$ & $1.138,06 \mathrm{~b}$ \\
\hline \multicolumn{5}{c}{ PSIM } \\
1 & $-238,83 \mathrm{~b}$ & $-172,67 \mathrm{a}$ & $1.046,38 \mathrm{a}$ & $1.049,94 \mathrm{a}$ \\
\hline
\end{tabular}

${ }^{(1)}$ Médias seguidas da mesma letra, na linha, não diferem entre si pelo LSMEANS $(\mathrm{P}>0,01)$.

como pelo resíduo foliar remanescente pós-pastejo. Embora os métodos tenham proporcionado estimativas semelhantes nas duas épocas, torna-se necessária a calibração a cada amostragem, de modo que as equações geradas sejam mais precisas e possibilitem a obtenção de valores confiáveis das estimativas.

\section{Conclusões}

1. O método do rendimento visual comparativo e o método do pastejo simulado são eficientes para estimar a forragem potencialmente consumível em pastagem de capim-elefante.

2. As estimativas obtidas pelo método do rendimento visual comparativo são mais confiáveis que as obtidas pelo método do pastejo simulado.

\section{Referências}

ABRAMIDES, P. L. G.; ALCÂNTARA， P. B.; STAFUZZA, J. A.; FOSCHINE, A.; DOWER, J. B. Estimativa da quantidade de forragem em pastagens de capins prostrados tropicais, através da medida da altura média da vegetação. Zootecnia, Nova Odessa, v. 20, n. 1, p. 17-41, 1982.

ABRAMIDES, P. L. G.; FRANZINI, W. Utilização de quatro variáveis, isoladas ou associadas, na estimativa de quantidade de forragem em duas pastagens de capins tropicais de hábito cespitoso. Zootecnia, Nova Odessa, v. 19, n. 2, p. 115-139, 1981.

AIKEN, G. E.; BRANSBY, D. I. Observer variability for disk meter measurements of forage mass. Agronomy Journal, Madison, v. 84, n. 4, p. 603-605, 1992. 
ALENCAR, C. A. B. Resultados obtidos em fazendas produtoras de leite em pastagens manejadas intensivamente. In: MARTINS, C. E.; CÓSER, A. C.; YAMAGUCHI, L. C. T.; MENDES, L. C. R.; LIMA, I. B.; SILVA, M. P.; PEDROSA, V. L. (Ed.). Gestão estratégica para o desenvolvimento da pecuária leiteira na região Campo das Vertentes. Juiz de Fora: Embrapa CNPGL/Cemig, 2002. p. 98-122.

AROEIRA, L. J. M.; LOPES, F. C. F.; DERESZ, F.; VERNEQUE, R. S.; MALDONADO VASQUEZ, H.; MATOS, L. L.; VITTORI, A. Pasture availability and dry matter intake of lactating crossbred cows grazing elephant grass (Pennisetum purpureum Schum.). Animal Feed Science and Technology, Amsterdam, v. 78, p. 313-324, 1999.

COELHO, R. W. Técnicas de estimativa de disponibilidade de forragem. São Carlos: Embrapa-Uepae de São Carlos, 1984. 28 p. (Circular Técnica, 2).

CÓSER, A. C.; MARTINS, C. E.; CARVALHO, C. A. B.; GERÔNIMO, O. J.; FREITAS, V. P.; SALVATI, J. A Avaliação de metodologias para a estimativa da disponibilidade de forragem em pastagem de capim-elefante. Revista Ciência e Agrotecnologia, Lavras, v. 26, n. 3, p. 589597,2002

CÓSER, A. C.; NASCIMENTO JUNIOR, D.; MARTINS, C. E.; VERNEQUE, R. S. Relação cobertura:peso em pastagens do planalto de Viçosa, MG. Revista da Sociedade Brasileira de Zootecnia, Viçosa, MG, v. 18, n. 1, p. 48-53, 1989.

CÓSER, A. C.; TEIXEIRA, F. V.; CAMPOS, O. F.; MARTINS, C. E. Utilização das co-variáveis altura da planta e cobertura do solo para a estimativa da forragem disponível em pastagem de capim-elefante. Revista Ciência e Agrotecnologia, Lavras, v. 24, n. 3, p. 804-814, 2000.

ESTRADA, C. L. H.; NASCIMENTO JUNIOR, D.; REGAZZI, A. J. Efeito do número e tamanho do quadrado nas estimativas pelo Botanal da composição botânica e disponibilidade de matéria seca de pastagens cultivadas.
Revista da Sociedade Brasileira de Zootecnia, Viçosa, MG, v. 20, n. 5, p. 483-493, 1991.

HAYDOCK, K. P.; SHAW, N. H. The comparative yield method for estimating dry matter yield of pasture. Australian Journal of Experimental Agriculture and Animal Husbandry, Melbourne, v. 15, n. 76, p. 663-670, 1975.

LOPES, R. S.; FONSECA, D. M.; CÓSER, A. C.; NASCIMENTO JUNIOR, D.; MARTINS, C. E.; OBEID, J. A. Avaliação de métodos para estimação da disponibilidade de forragem em pastagem de capim-elefante. Revista Brasileira de Zootecnia, Viçosa, MG, v. 29, n. 1, p. 40-47, 2000.

MARTINS, C. E.; CÓSER,A. C.; DERESZ, F. Manejo de solo e água em sistemas intensivos de produção de leite a pasto. In: MARTINS, C. E.; CÓSER, A. C.; YAMAGUCHI, L. C. T.; MENDES, L. C. R.; LIMA, I. B.; SILVA, M. P.; PEDROSA, V. L. (Ed.). Gestão estratégica para o desenvolvimento da pecuária leiteira na região Campo das Vertentes. Juiz de Fora: Embrapa CNPGL/Cemig, 2002. p. 135-160.

MORLEY, F. H. W.; BENNETT, D.; CLARK, K. W. The estimation of pasture yield in large grazing experiments. CSIRO Field Station Record, Brisbane, v. 3, n. 2, p. 43-47, 1964.

O'ROURKE, P. K.; McCOSKER, T. H.; TEITZEL, J. K.; STEPHENSON, H. P.; WILSON, R. J. Application and appraisal of a visual estimation technique for composition and yield sampling of grasslegume pastures in the wet tropics of north eastern Australia. Australian Journal of Experimental Agriculture and Animal Husbandry, Melbourne, v. 24, n. 127, p. 535-542, 1984.

SAS INSTITUTE (Cary, Estados Unidos). SAS user's guide: statistics 8.2. Cary, 2001. 1028 p.

THOMSON, N. A. Techniques available for assessing pasture. In: DAIRY farming annual. New Zealand: Massey University, 1986. p. 113-121. 